\title{
CircPSMC3 suppresses the proliferation and metastasis of gastric cancer by acting as a competitive endogenous RNA through sponging miR-296-5p
} (1) CrossMark

Dawei Rong ${ }^{1+}$, Chen $\mathrm{Lu}^{1+}$, Betty Zhang ${ }^{2}$, Kai Fu', Shuli Zhao ${ }^{3^{*}}$, Weiwei Tang ${ }^{1 *}$ and Hongyong $70^{1 *}$

\section{Abstract}

Background: Circular RNAs (circRNAs) are a class of non-coding RNAs with a 100 tructure, but its functions remain largely unknown. Growing evidence has revealed that circRNAs play a striking le retional RNAs in the progression of malignant disease. However, the precise role of circRNAs in gastn ancer (GC) remains unclear.

Methods: CircRNAs were determined by human circRNA array analysis polymerase reaction. Luciferase reporter, RNA pull down, and fluorescende in s, u hybridization assays were employed to test the interaction between circPSMC3 and miR-296-5p. Ectopic over-expression and siRNA-mediated knockdown of circPSMC3, proliferation, migration and invasion in vitro, nd is vo experiment of metastasis were used to evaluate the function of circPSMC3.

Results: CircPSMC3 rather than liner PSMC3 mRNA was down-1 alated in GC tissues, corresponding plasmas from GC patients as well as GC cell lines compared to normar nals. 'Ower circPSMC3 expression in GC patients was correlated with higher TNM stage and shorter overall sun val. Ove, nession of circPSMC3 and miR-296-5p inhibitor could inhibit the tumorigenesis of gastric cancer cells in ivo d vitro whereas co-transfection of circPSMC3 and miRNA-296-5p could counteract this effect. Importantly demo rated that circPSMC3 could act as a sponge of miR-296-5p to regulate the expression of Phosphatas and Tensin Homolog (PTEN), and further suppress the tumorigenesis of gastric cancer cells.

Conclusion: Our study reveals that circis es can serve as a novel potential circulating biomarker for detection of GC. CircPSMC3 participates in progress gastric cancer by sponging miRNA-296-5p with PTEN, providing a new insight into the treatment of gactric cancer.

Keywords: Gastric \& 2 ,

\section{Introductior}

Gastric caner (GC) the fifth most common cancer in the wo 1 a)d the third most common cause of cancer death wo, vide $[1]$. It tends to metastasize into neighborn. tissu and organs through lymph nodes and

*Corm Jndence: shulizhao79@163.com; 1243773473twww@sina.com; caohorgy6167@163.com

${ }^{\dagger}$ Dawei Rong and Chen Lu contributed equally to this work.

${ }^{3}$ Department of General Clinical Research Center, Nanjing First Hospital, Nanjing Medical University, Nanjing, Jiangsu, China

${ }^{1}$ Department of General Surgery, Nanjing First Hospital, Nanjing Medical University, Nanjing, Jiangsu, China

Full list of author information is available at the end of the article generate more cancer cells through the blood [2]. Although there have been many advancements in the diagnosis and treatment of GC, recurrence and metastasis are still occurring at high rates $[3,4]$. Improvements in clinical care for these patients are limited by the lack of clarity surrounding the molecular mechanism in GC development [5]. Thus, it is urgently necessary to explore new potential biomarkers and their molecular mechanisms to better understand the pathophysiology of gastric malignancies.

Circular RNAs (circRNAs) are a new class of endogenous non-coding RNAs characterized by covalently closed 
loops without $5^{\prime}$ to $3^{\prime}$ polar or polyadenylation tails [6]. Previous studies have shown that circRNAs are formed by the back-splicing of pre-mRNA transcripts from genes with five different forms [7]. CircRNAs are stable, conserved and abundant in various cancer tissues or cell lines, as tissue/developmental stage-specific circRNAs are usually notable $[8,9]$. Kuei-Yang Hsiao et al. found that circRNA CCDC66 could promote the progression and metastasis of colon cancer [10]. Studies on the molecular mechanism of circRNAs indicate that circRNAs can act as a competitive endogenous RNA (ceRNA) to regulate downstream genes associated with diseases by binding to miRNAs [11-13]. Xuetao Cao et al. found that circMTO1 might regulate the progression of hepatocellular carcinoma (HCC) by regulating the expression of p21 as a sponge of oncogenic miR-9, which can be used as a potential target for HCC therapy and a prognostic indicator for low patient survival [14]. Zhenyu Zhong et al. indicated that circMYLK could act as ceRNA of miR-29a, further promoting the progression of Epithelial-Mesenchymal Transition (EMT) in bladder cancer by activating VEGFA/VEGFR2 and Ras/ERK signaling pathways [15].

MicroRNAs (miRNAs), as a conserved small regulatory non-coding RNA, have been demonstrated to involve many biological functions in different disfases [16]. Many studies have reported that miRNAs ca go late by different circRNAs and lncRNAs to fur titer $r$ late gene expression [17-19]. Yawei Li et and tha circHIPK3, which contains two key binding tes of miR-558, directly regulates miR-558 unction to 1 hibit heparanase (HPSE) expression. Th $r$ findings suggest that circHIPK3 acts as a "miRNA sp e" ard identifies circHIPK3 as a new therapeut arget for patients with bladder cancer [20].

In this study, based or resul s of circRNA arrays, we identified a circular $D$ te med circPSMC3 derived from PSMC3 gene. Cir $1 \mathrm{SM}$ was down-regulated in tissues, corresponding $\mathrm{A}$ mas fro $1 \mathrm{GC}$ patients as well as GC cell lines and coul t as a sponge of miRNA-296-5p to regulate the expressio, of Phosphatase and Tensin Homo$\log (\mathrm{PT}$ and yrther suppress the tumorigenesis of GC cell- -ur dirgs provide insight into the treatment of stri cance and reveal a novel potential circulating bio$\mathrm{n}$. er ru detection of GC.

\section{Materials and methods}

\section{Cell cultures and patient tissues}

Human gastric cancer cell lines (BGC823, MGC803, SGC7901, AGS, and MKN45) were purchased from Shanghai Institutes for Biological Sciences, China. The human gastric epithelial cell line GES-1 was obtained from the Cancer Institute and Hospital of the Chinese Academy of Medical Sciences (Beijing, China). All cell lines were cultured in RPMI 1640 medium (Gibco, Carlsbad, CA, USA) supplemented with 10\% fetal bovine serum (Gibco, Vienna, Austria) and in a humidified incubator containing $5 \% \mathrm{CO}_{2}$ at $37^{\circ} \mathrm{C}$.

One hundred and-six samples of GC tissues were matched to adjacent normal tissues and $10 \mathrm{~m} /$ preoperative blood venous blood were collected rom the GC patients treated in Department of General

Nanjing Hospital, Nanjing Medical University a ig 2013 to 2016 in accordance with the 'sinki Declaration. Twenty-one samples of 10 111 no al venous blood were randomly obtained rom the $5,-90$ years old individuals without any unde ing diseases in physical examination center or an) nospital during 2015 to 2016. All these pecim were frozen in liquid nitrogen and stably s $\mathrm{O}_{1} \quad$ at $-80{ }^{\circ} \mathrm{C}$ until RNA extraction. Histological and pa logical diagnoses of these specimens wer $\mathrm{CO}_{1}$ irmed and classified by two experienced clinica ists. Informed consent from these patients ha een obtained before specimen collection. 1 rojec was approved by the Ethics Committee of $(\mathrm{a} / \mathrm{i}) \mathrm{n}, \mathrm{s}$ Medical University.

\section{Puan tative reverse transcription polymerase reaction} T.PCR)

Aecording to the manufacturer's protocol, total RNAs from tissues, plasma and cells were isolated by using TRIzol reagent (Invitrogen, CA, USA). For circRNA and mRNA, cDNA was synthesized by using reverse transcription kit (Takara, Otsu, Japan) and for miRNA, total RNAs were reversed using RiboBio reverse transcription kit (Guangzhou, China). Quantification of mRNA and circular RNA was performed by using a SYBR Green PCR Kit (Takara, Otsu, Japan), and miRNA PCR was performed by using a SYBR Green PCR Kit (RiboBio,Guangzhou, China). All primer sequences were designed and synthesized by Genery (Nanjing, China). CircPSMC3 expression level was detected using the following primer pair: 5'-GTTTAGGGTCCCTGCCCTTTG-3' (Forward, or F) and 5'-GTGTTGGGCTGGAAGCCATC-3' (Reverse, or $\mathrm{R})$. The primer pair of PSMC3 is 5' - AGACGCTGC CCACAGAGTATG $-3^{\prime}$ (F) and 5'- CTTTTGGAG GTTGGATCCCC-3' (R). GAPDH was used to normalize the mRNA and circRNA expression levels and U6 was used to normalize the miRNA expression levels before calculation.

\section{RNase R treatment}

Total RNA $(10 \mu \mathrm{g})$ of gastric cancer cell lines was mixed with $40 \mathrm{U}$ RNase $\mathrm{R}$ at $37^{\circ} \mathrm{C}$ for $2 \mathrm{~h}$. To assess the stability of circPSMC3 and line PSMC3 mRNA, the expression levels were determined by using qRT-PCR. 


\section{Oligonucleotide transfection}

Si-circPSMC3, miRNA-296-5p mimic, miRNA-296-5p inhibitor and their related control oligonucleotide were designed and synthesized by RiboBio (Guangzhou, China). The sequence of siRNA:siRNA-1:TAGGGTCCCTGCCC TTTGA, siRNA-2:GGGTCCCTGCCCTTTGACA, siRN A-3: TCCCTGCCCTTTGACAGTG. All transfections were performed by the final concentration of $60 \mathrm{nM}$ of miRNA mimics and $100 \mathrm{nM}$ of miRNA inhibitor and si-circPSMC3. Lipofectamine 2000 reagent (Invitrogen) was used as transfection medium.

\section{Plasmids construction and stable transfection}

To isolate stable human gastric cancer cells over-expressing circPSMC3, circPSMC3 cDNA was synthesized cloned into pcD-ciR and pcDNA3 vector and lentivirus (Hanheng, Shanghai, China). According to the manufacturer's instructions, human gastric cancer cell lines, MGC803 and BGC823 were infected with lentivirus at a multiplicity of infection of 50 . All cell lines were followed by selection with $2 \mu \mathrm{g} / \mathrm{mL}$ puromycin for 2 weeks.

\section{Luciferase reporter assay}

The wild-type and mutant fragments in 3 '-UTR of circPSMC3 related with miRNA-296-5p binding site vere designed, synthesized and inserted into pGL3-bas ve tors (Realgene, Nanjing, China), then pGL3-basic veo is and miRNA-296-5p mimics or inhibitor of rcPSMC, overexpressing lentivirus were co-transfected to $-\mathrm{T}$ cell respectively. After $48 \mathrm{~h}$, according to the manufac,urer's instructions, luciferase activity in co-t nsfected cells were collected and detected by the dua ciferse reporter assay system (Promega).

\section{Biotin-coupled probe RN ull do vn assay}

Biotin-coupled prob. RN null down assay was performed. To pull down $\mathrm{t}$ mikNA by circRNA, MGC803 and BGC823 wit transfect a with miRNA-296-5p mimics were lysed and in bated with Biotin-coupled probe of circPSM 33 which wa pre-bound on magnetic beads. For $2 \mathrm{~h}$, targ R A vas pulled by the RNeasy Mini Kit (QIAGF Ge ny). Then the pull-down product was trac ed, re ersed and placed through q-PCR. To pull a 1 cin circRNA by miRNA, MGC803 and BGC823 with ircPSMC3 over-expression and Biotin-coupled probe of miRNA-296-5p were processed through the same protocol.

\section{Fluorescence in situ hybridization (FISH)}

The fluorescence in situ hybridization assay was performed to detect the presence of circPSMC3 and miRNA-296-5p by using Fluorescence in Situ Hybridization Kit (RiboBio, Guangzhou, China). CircPSMC3 was captured with
Cy5-labeled probe and miRNA-296-5p was captured with Cy3-labeled probe respectively. After prehybridization, circPSMC3 probe and miRNA-296-5p probe were hybridized in prepared hybridization buffer in MGC803 cells. Nuclei were marked by staining with 4,6-diamidino-2-phenylindole (DAPI). Confocal microscopy wa used to better visualize the presence of circPSN C3 and miRNA-296-5p.

\section{Cell counting kit-8 proliferation assay ind thyny,-20-} deoxyuridine (EdU) incorporation a say

GC cells were seeded in 96 we with the density of 4000 cells per well. Seeded ${ }^{1 / s}$ h troated with $10 \mu \mathrm{l}$ of CCK8 solution (Ribobi, Gu. zhou, China) after cultured at $0 \mathrm{~h}, 24 \mathrm{~h}, 48 \mathrm{~b}, \mathrm{~h}, 96 \mathrm{~h}$, espectively. Then the absorbance of cells an eac ime was analyzed at $450 \mathrm{nM}$ by microplate re accord, g to the manufacturer's instructions (Sy oy RinTek, Winooski, VT, USA). The EdU assay was $P$ formed to assess the proliferation of cells by ing a Ce-Light EdU DNA Cell Proliferation Kit (Ribobio, ngzhou, China). GC cells were plated in 24 wells and were cultured for $24 \mathrm{~h}$. These two cell were fixed using $4 \%$ paraformaldehyde after incubatio with $50 \mathrm{mM}$ EdU solution for $2 \mathrm{~h}$. Then according th. manufacturer's protocol, cell lines were sealed $w_{1}$ a Apollo Dye Solution and Hoechst 33342 in order. The EdU cell lines were photographed and counted under an Olympus FSX100 microscope (Olympus, Tokyo, Japan).

\section{Transwell migration and invasion assays}

For this assay, according to the manufacturer's protocol, GC cells were seeded in upper chambers with $200 \mu \mathrm{l}$ of serum-free medium. The transwell chamber (Corning, NY, USA) was paved with matrigel mix (BD Biosciences, San Jose, CA, USA) for invasion assays and without matrigel mix for migration assays. The bottom chamber was filled with medium and 10\% FBS as a gastric cancer cell chemoattractant. After incubation for $24 \mathrm{~h}$, the upper chambers were fixed and then stained by crystal violet (Kaigen, Nanjing, China) for $15 \mathrm{~min}$. For visualization, the cell lines were photographed and counted in different five fields.

\section{Cell apoptosis assays}

GC cells were stained with FITC and PI from the Annexin V-FITC/Propidium Iodide (PI) Apoptosis Detection Kit (BD Biosciences \#556547). FACScan (BD Biosciences, San Jose, CA, USA) was used to analysis stained cells and all apoptosis data of different cell lines was analyzed by Flowjo V10 software (Tree Star, San Francisco, CA, USA). 


\section{Western blot}

Cells were lysed in RIPA lysis buffer (RIPA, Beyotime, China). The protein was prepared and quantified by bicinchoninic acid (BCA) analysis (Beyotime, China). The same amounts of protein were extracted by $10 \%$ SDS-PAGE and transferred onto a PVDF membrane (Millipore, Schwalbach, Germany). The blocked protein with $5 \%$ skim milk powder was incubated with primary antibody anti-PTEN (\#3285, Cell Signaling Technology), anti-YYM (\#66281-1-Ig, Proteintech), anti-GAPDH (\#ab181602, Abcam).at $4{ }^{\circ} \mathrm{C}$ for $12 \mathrm{~h}$.Then the prepared membranes were incubated with secondary antibody (1:5000) for $2 \mathrm{~h}$. Finally, the blots were detected by enhanced chemiluminescence kit (Pierce, Waltham, MA, USA) and related data was analyzed by Image Lab Software.

\section{Xenografts in mice}

The animal assay was approved by the animal management committee of Nanjing Medical University, and all experimental procedures and animal care were in accordance with the institutional ethics guidelines for animal experiments. To create the xenograft tumor model, 20 5-week-old male nude mice were separated randomly into over-circPSMC3 group and NC group ( $n=10$ for each group). About $1 \times 10^{7}$ circPSMC3 over-expr ssing MGC803 cells were subcutaneously injected into th. illa of the nude mice respectively. The vo $1 \mathrm{me}$ of injected nude mice was measured every 3 day $v$ using digital calipers. After 35 days, all inizced nua mice were sacrificed, excised tumor weig its were measured and tumor tissues were studied $\mathrm{b}$ hematoxylin and eosin (H\&E) and IHC staining. To $P$.ce the nude mice metastasis model, $205-n+1 d$ male nude mice were separated randomlv into over circPSMC3 group and NC group $\left(n=10 \mathrm{to}\right.$ each group). About $2 \times 10^{6}$ circPSMC3 over-expro ir C803 cells were tail-vein injected into 20 -week- male BALB/c nude mice respectively. $\mathrm{Si}$ wt later, the nude mice were sacrificed; pulmonary metasta nodules were counted by three patholo ists after the lungs were removed by experienced su ons. The lungs removed were studied by usinc ema ylin-eosin staining.

\section{Statı.ál analysis}

The analyses were mainly performed by using SPSS 19.0 (IBM, SPSS, and Chicago, IL, USA) and $p$-value $<0.05$ was demarcated to be statistically significant. Comparison of continuous data was analyzed using an independent $t$-test between the two groups, whereas categorical data was analyzed by the chi-square test. Kaplan-Meier method was mainly used to assess the survival rate and analyzed by using log rank test.

\section{Results}

CircPSMC3 is significantly down regulated in gastric cancer and associated with poor prognosis

To investigate the role of circRNAs in the development of gastric cancer, the circRNA expression signatures in gastric cancer plasma were explored by using cir RNA microarray analysis using plasma samples fror $10 \mathrm{GC}$ patients, including 5 patients with no lymph metastasis and the other 5 patients with lymph node tasis, and 5 normal individuals. The res show dhat 6405 circRNAs in lymph node mccastas oroup and normal group (Fig. 1a) and 3443 r reRNA in $1 / \mathrm{mph}$ node metastasis group and no lymph de metastasis group (Fig. 1b) were significantly an ald change $>2.0$ and $P<0.05$. GO pathw $y$ analy suggest that these differentially expressed $C_{1}$. NAs a re relevant to several vital physiological process molecular functions, and critical signalin, pa ways in two groups (Fig. 1c-d).

We selected 3 circRNAs based on the multiple fold differe in circRNA microarray and then verified $t$.ndingo in a small sample of plasmas by using $\mathrm{qRT}-\mathrm{P}$-ly as well as the structure, length, and source of cirRNA (Additional file 1: Figure S1a). Results Sil d that a novel circRNA named circPSMC3, which has ver been reported in previous literature, has a vif/cantly lower expression in GC plasmas compared to normal controls, which was then picked out for further study. The spliced mature sequence length of circPSMC3 derived from the PSMC3 gene is 502 bp according to circbase database (http://www.circbase.org/) (Fig. 1e) and circPSMC3 is derived from exons, while no other subtypes of circPSMC3 are found. The stability of circPSMC3 was evaluated and results showed that circPSMC3 harbors a loop structure with the resistance to digestion by RNase R (Fig. 1f), while PSMC3 mRNA could be degraded by RNase R (Fig. 1g).

Given that the tremendous diagnostic and therapeutic role of circRNAs in GC, we explored the clinical value of circPSMC3 by detecting its expression in GC samples. Results indicated circPSMC3 had significantly lower expression in GC plasmas (Fig. 1h), tissues (Fig. 1i) and cells (Fig. 1j) compared to normal controls. In addition, circPSMC3 expression was lower in preoperative blood from GC patients with lymph node metastasis compared to those patients without lymph node metastasis (Fig. 1h). Clinicopathological features showed that down-expression of circPSMC3 was negatively associated with TNM stage (Table $1, P=0.000$ ) and lymphatic metastasis (Table 1, $P=0.021)$. However, circPSMC3wa not associated with the gender, age, size, or histological grade. Furthermore, the area under the ROC curve (AUC) of circPSMC3 in distinguishing GC plasmas and normal ones was 0.9326 (Fig. 1k) and the cut-off value was -9.965 with the sensitivity of $85.85 \%$ and specificity of $95.24 \%$. Kaplan-Meier 


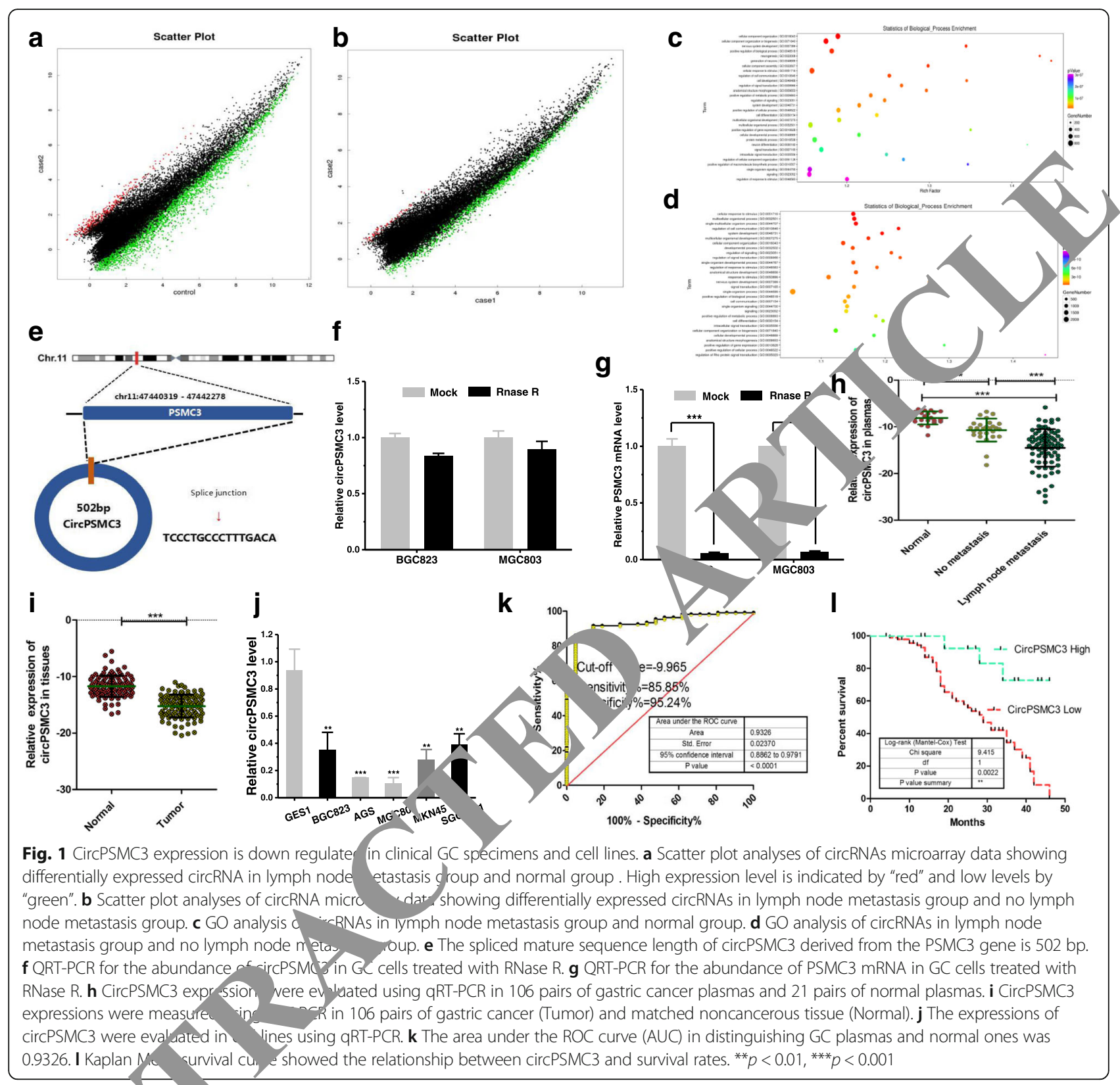

overal v clurve revealed that patients with lower circ ${ }^{\mathrm{BC}} \mathrm{MC}$ vpression showed a reduced overall survival me (ig. 11).

\section{Circt 'C3 plays a suppression role in gastric cancer cells in vitro}

To evaluate the role of circPSMC3 in GC cells, three siRNAs against circPSMC3 were designed to silence circPSMC3 without influencing PSMC3 mRNA level in BGC823 and SGC7901 cells (Additional file 1: Figure S1b-1d) and finally si-circPSMC3\#1 was chosen for the following experiment with its high inhibitory efficiency. The circular transcript expression vector circPSMC3 was successfully constructed in MGC803 and AGS cells (Fig. 2a), as it could increase circPSMC3 expression level rather than PSMC3 mRNA (Additional file 1: Figure S1e-1f). The results of CCK-8 and EdU assay showed that si-circPSMC3 could promote cell proliferation in BGC823 and SGC7901 cell lines, whereas over-expression of circPSMC3 (named circ-PSMC3) might inhibit cell proliferation in MGC823 and AGS cell lines (Fig. 2b-c). Wound healing assay showed that silencing of circPSMC3 significantly increased the cell mobility, while over-expression of circPSMC3 might inhibit the cell mobility (Fig. 2d). The result of cell invasion assay showed that down regulation of circPSMC3 significantly increased cell invasion and 
Table 1 Correlations between circPSMC3 expression in plasmas and clinical characteristics in GC patients

\begin{tabular}{|c|c|c|c|c|}
\hline \multirow[t]{2}{*}{ characteristic } & \multirow[t]{2}{*}{ case } & \multicolumn{2}{|c|}{ circPSMC3 expression } & \multirow{2}{*}{$\begin{array}{l}p \\
\text { value }\end{array}$} \\
\hline & & low & high & \\
\hline All cases & 106 & 91 & 15 & \\
\hline Age (yeas) & & & & 0.530 \\
\hline$<65$ & 40 & 34 & 6 & \\
\hline$\geq 65$ & 66 & 57 & 9 & \\
\hline Gender & & & & 0.250 \\
\hline Female & 37 & 34 & 3 & \\
\hline Male & 69 & 57 & 12 & \\
\hline Tumor size (cm) & & & & 0.266 \\
\hline$<5$ & 42 & 34 & 8 & \\
\hline$\geq 5$ & 64 & 57 & 7 & \\
\hline Histological grade & & & & 0.309 \\
\hline High & 23 & 18 & 5 & \\
\hline Middle-low & 83 & 73 & 10 & \\
\hline Lymph node metastasis & & & & $0.021^{\prime}$ \\
\hline Negative & 27 & 19 & 8 & \\
\hline Positive & 78 & 72 & 7 & \\
\hline TNM stage & & & & $0.000^{\circ}$ \\
\hline$|-| \mid$ & 38 & 24 & 14 & \\
\hline III-IV & 68 & 67 & 1 & \\
\hline
\end{tabular}

indicates $P<0.05$

over-expression of circPSMC3 exhibited the (Fig. 2e).

\section{CircPSMC3 directly binds to miR-296- and suppresses} miR-296-5p activity

Given that circRNAs could bin different miRNAs and regulate downstream genes, we to n that circPSMC3 possessed a complemen sequence to miR-296-5p seed region by bioinforma an trcis t/rough Circinteractome database (https://ercin actome.nia.nih.gov/). To confirm the web, predict $\Lambda$, the biotin-coupled probe pull-down assay $\mathrm{w}$ performed and the results showed miR-296 op and c1 cPSMC3 were detected in the circPSin gro (Fig. ). urthermore, the result of FISH indicated at a CPSM 3 was co-localized with miR-296-5p in the c) rasm of MGC803 cell lines (Fig. 3b).

In dition, luciferase reporters with either the wild type circPSMC3 sequence (WT) or the sequence with mutated binding sites of miR-296-5p (Mut) into the 3 UTR of renilla luciferase showed that miR-296-5p over-expression could significantly reduce the luciferase activities of WT reporter rather than mutant one (Fig. 3c). QRT-PCR further confirmed that circPSMC3 knockdown could increase the miR-296-5p level and circ-PSMC3 had an opposite role in GC cell lines (Fig. 3d). However, miR-296-5p failed to influence circPSMC3 level (Fig. 3e). Collectively, these revealed that circPSMC3 could bind to miR-296-5p to further regulate its expression level.

\section{MiR-296-5p targets PTEN and promotes the proliferation} and invasion of gastric cancer cells

According to miRanda database prediction (http:// mirdb.org/), miR-296-5p could target PTEN n. 'A 3 ' UTR with a high score.

This interaction was confirmed by per rming 'uciferase reporter assays. The result show th the over-expression of miR-296-5p ould signi) cantly reduce the activity of a luciferase porter compared to miR-NC and the inhibition the 296-5p may evidently increase the luriterase tivity compared with inh-NC with wild tyre JEN sequence (WT), however, these effect disappeared miR-296-5p (Nuı Fig. 4d). The knockdown or over-expressio $\quad$ miR-296-5p was successfully established (Ada anal file 1: Figure S1g-1 h). We found tha R-296-5p over-expression significantly reduced the P $1 \mathrm{~L}, \mathrm{mRNA}$ levels in GC cells (Fig. 4b). Western biot further confirmed that transfection of mu 96-5p mimics could reduce PTEN expression (Fig. c). These results showed that miR-296-5p could ratively regulate the expression of PTEN.

he role of miR-296-5p on the GC cell proliferation, viability, invasion, and migration was evaluated. The results indicated that over-expression of miR-296-5p promoted the proliferation (Fig. 4d-e), migration (Fig. 4f) and invasion (Fig. 4g) of GC cells. However, down expression of miR-296-5p might exert an opposite effect (Fig. 4d). These results suggest that miR-296-5p could target PTEN and further promote the development of GC partially.

CircPSMC3 suppresses the proliferation and invasion of gastric cancer by sponging miR-296-5p to regulate PTEN

In order to further explore the interaction among circPSMC3, miR-296-5p and PTEN, we performed luciferase reporter assays. The data showed that the over-expression of circPSMC3 could significantly increase the activity of a luciferase reporter, however, the co-transfection of circPSMC3 and miR-296-5p may eliminate this effect with wild type PTEN sequence (WT), and these effects disappeared with mutated binding sites of miR-296-5p (Mut)(Fig. 5a). Moreover, we found that circ-PSMC3 significantly increased the PTEN mRNA levels, whereas co-transfection of circ-PSMC3 and miR-296-5p may cancel out this effect in MGC803 and AGS cells (Fig. 5b). Western blot showed that circ-PSMC3 could promote PTEN expression, while co-transfection of circ-PSMC3 and miR-296-5p had no effect on PTEN level (Fig. 5c). These results demonstrated that circPSMC3 


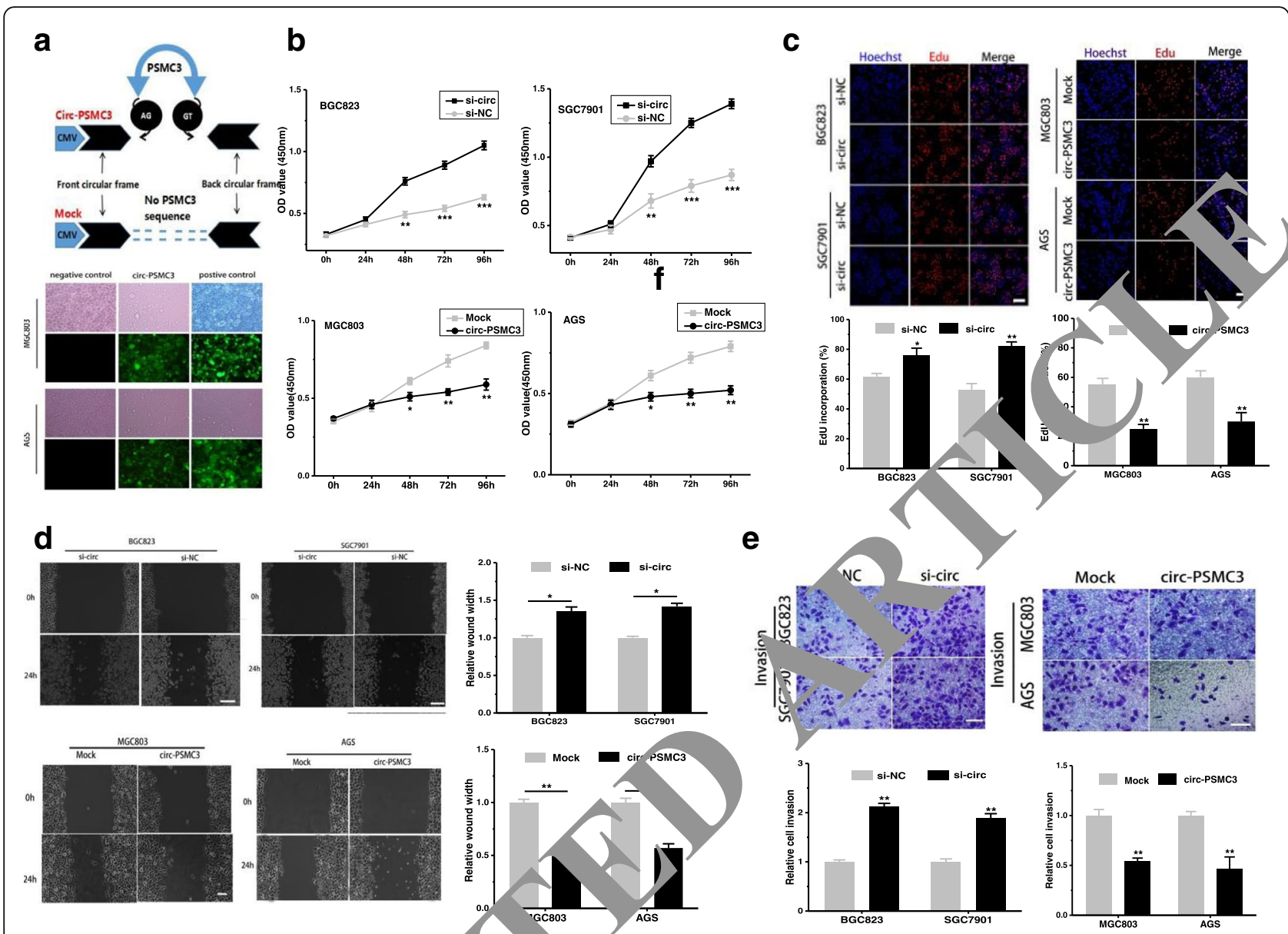

Fig. 2 CircPSMC3 produces suppression effects on rastric ca cells. a The circular transcript expression vector circPSMC3 was constructed. $\mathbf{b}$ The growth curves of cells were measured afte transfection w, n circPSMC3 vector or Mock vector or si-circ or si-NC by using CCK-8 assays. c EdU assays of GC cells transfected with contr or cirCPSMC3 siRNAs or circPSMC3 vector or Mock were performed to evaluate cell proliferation. $\mathbf{d}$ Cell motility was examined in cells transfected ith circPsMC3 vector or Mock vector or si-circ or si-NC by wound healing assay. e Cell invasion assays were performed in cells transfected with or circPSMC3 siRNAs or circPSMC3 vector or Mock. Data indicate mean \pm SD of at least three independent experiments. ${ }^{*} p<0 \quad{ }^{*} \times 0.01,{ }^{* * *} p<0.001$, Scale bar, $100 \mathrm{~mm}$

could regulate PTEN pre -inn by acting as a competing endogenous RNA to sp ge miR-296-5p. Results of the malignant beh? of circ1 MC3 and miR-296-5p on GC cell proliferation, vi lity, invasion migration and metastasis indicred that the circ-PSMC3 could inhibit proliferation, in $i$ and migration of GC cells. However, co- $^{+}$nsfec $n$ er circPSMC3 and miR-296-5p may counract his effect (Fig. 5d-h). These results of experiments Su esteu nat CircPSMC3 suppresses the proliferation, invasio and migration of gastric cancer cells by sponging miR-296-5p to regulate PTEN.

\section{Circ-PSMC3 inhibits the growth and metastasis of gastric cancer in vivo}

To explore the association between circPSMC3 and the growth as well as metastasis of gastric cancer in vivo, MGC803 cells transfected with circPSMC3 and GFP was injected into nude mice to established xenograft tumor model and metastasis nude mice model (Fig. 6a). In xenograft tumor model, we found that over expressing of circPSMC3 generated a negative effect on the volume of nude mice (Fig. 6b) as well as the weight (Fig. 6c). Ectopic over-expression of circPSMC3 inhibited metastasis in the lung compared to normal expression of circPSMC3 (Fig. 6d). Taken together, we illustrated that the over-expression of circPSMC3 could inhibit the proliferation, invasion and metastasis of GC cells and then suppress the progression of GC by sponging miR-296-5p to regulate PTEN expression (Fig. 6e).

\section{Discussion}

Deep sequencing combined with novel bioinformatics approaches led to the discovery that a significant portion of the human transcriptome is spliced into RNA loops [21]. In the last few years, several research groups have published interesting results, shedding light on the 


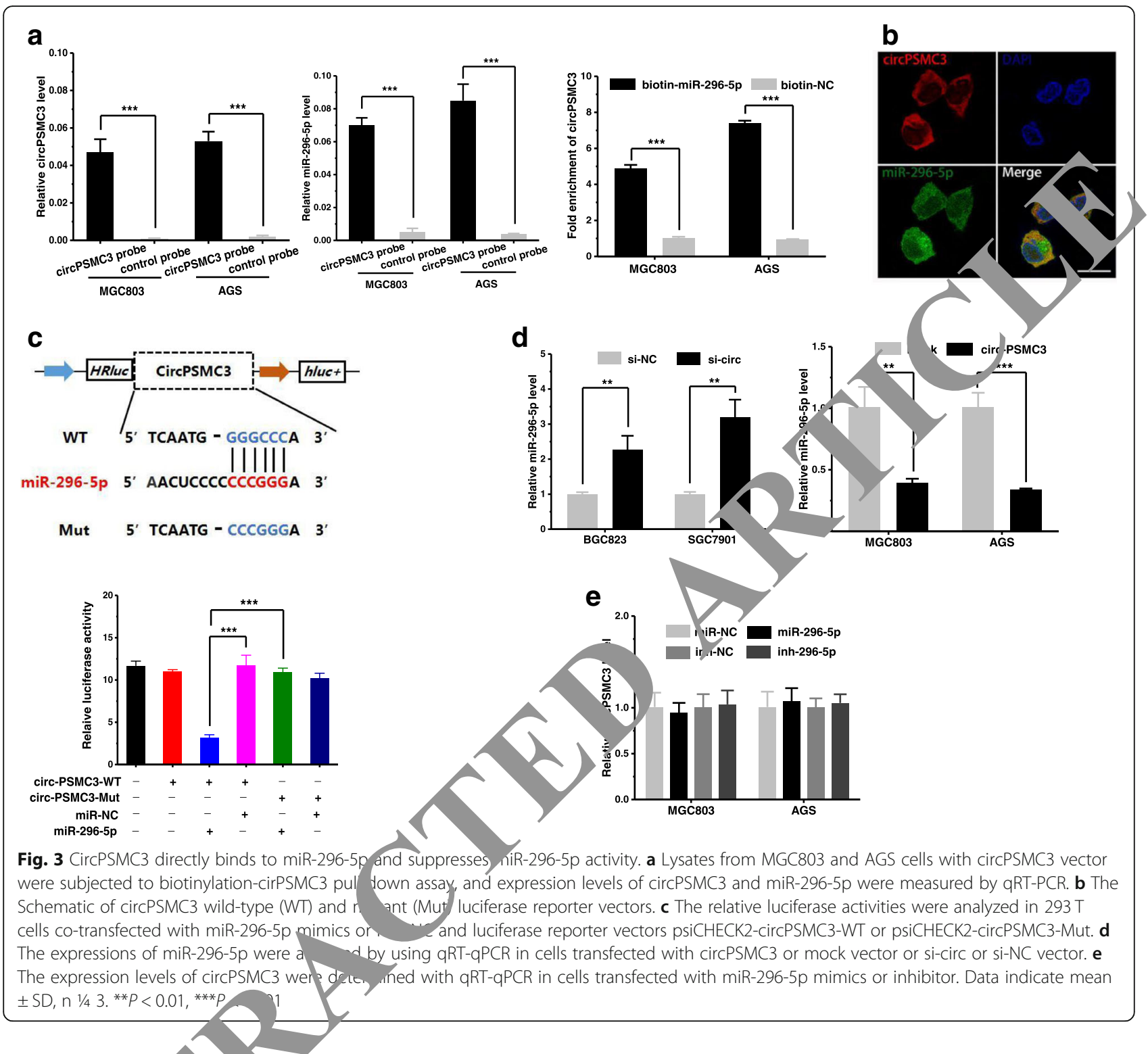

biogenesis of $\mathrm{C}$ NAs an possible mechanisms involving them [22]. So of these discoveries have shown that circrNAs are vyy stable, abundant and present a tissue $s_{1}$ if_ex ression pattern [23].

Ir-ur s. $\mathrm{V}$, we confirmed that circPSMC3 was sigifica tly loy er expressed in GC plasmas, tissues and co compared to normal controls. Clinicopathological featu illustrated that down expression of circPSMC3 was negatively associated with TNM stage and lymphatic metastasis, with a reduced overall survival time for GC patients. More and more studies have explored the relationship between circRNAs and the development of gastric cancer from a clinical perspective and investigated its application as a tumor biomarker in clinic. For example, Xie $\mathrm{Y}$ et al. detected the expression levels of hsa_circ_0074362 in 127 gastric cancer tissues and paired adjacent normal tissues by quantitative reverse transcription-polymerase chain reaction. Results showed hsa_circ_0074362 levels were significantly down regulated in gastric cancer tissues, gastritis tissues and gastric cancer cell lines and were associated with lymphatic metastasis, which may be a potential biomarker of gastric cancer [24]. However, most of the studies only detect the expression of circRNAs from cancer tissues and adjacent tissue. Only a small number of studies detect the expression of circRNAs from preoperative blood in GC patients and the sample size is small. The results of our study make circPSMC3 an ideal noninvasive biomarker for the diagnosis and prognosis of gastric cancer.

We demonstrated that miR-296-5p targets PTEN and promotes the proliferation and invasion of GC cells. Current studies show that miR-296-5p plays a role in 


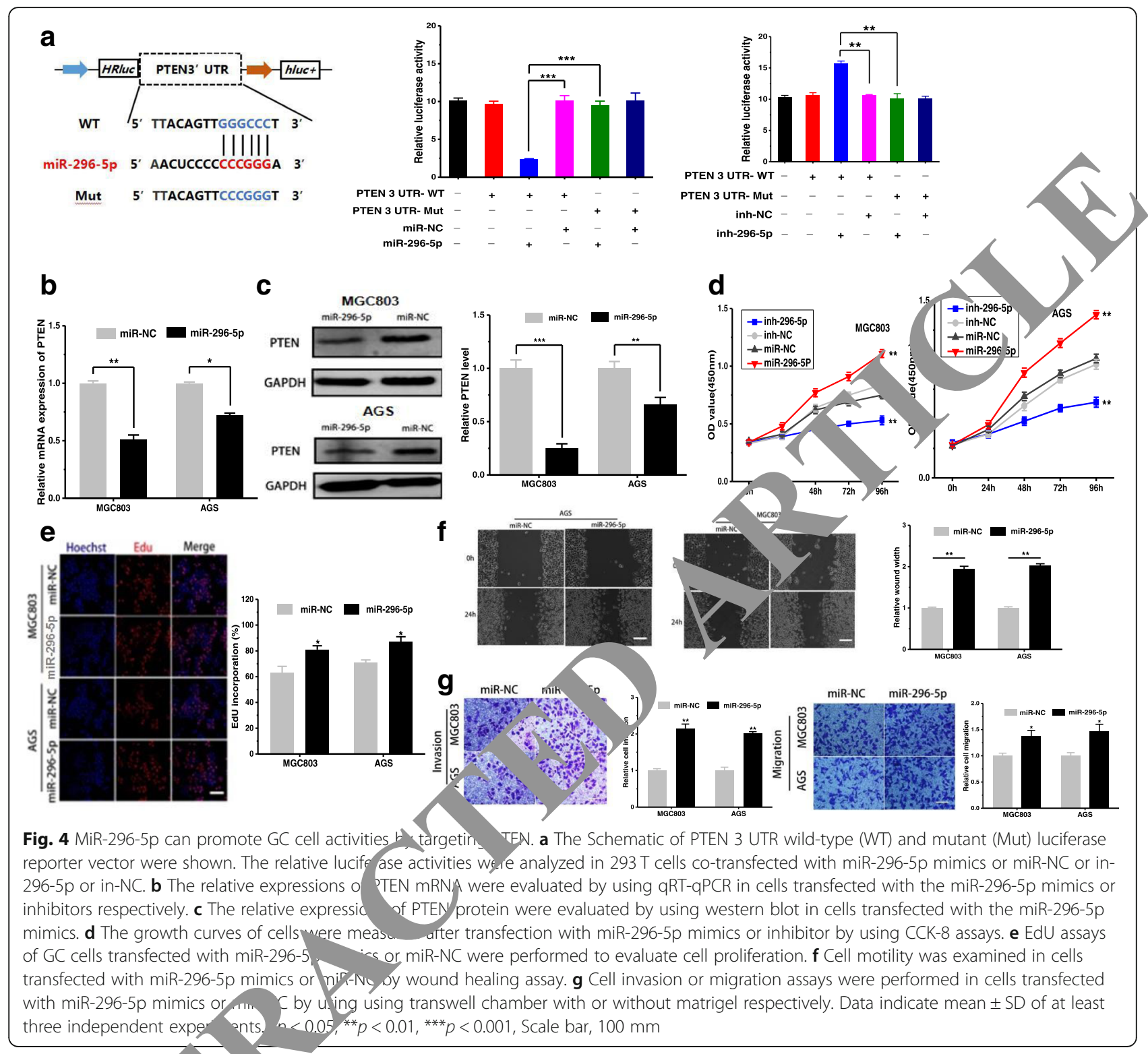

cancers. For exa le, Lee $\mathrm{H}$ et al. observed that miR-296 $\mathrm{p}$ promote, the invasion of various glioblastomas a con results obtained from Ago2 immunopre-itati and luciferase assays, they found that iR- 6-5p uownregulated CASP8 and NGFR through $a_{1}$ imuraction between seed sequence of the miRNA and YTR of the target mRNA. Collectively, their results implicated miR-296-5p as a potential cause of invasiveness in cancer and identifies miR-296-5p as a promising therapeutic target for glioblastomas [25]. Maia D reported miR-296-5p expression is associated with resistance to radiotherapy and tumor recurrence in early stage laryngeal squamous cell carcinoma, showing the feasibility of this marker as a novel prognostic factor for this malignancy. Furthermore, miR-296-5p expression could be helpful in the identification of tumors resistant to radiotherapy, thus informing treatment plans [26]. Interestingly, Lee $\mathrm{KH}$ reported that miR-296-5p has a tumor-suppressive role by targeting Pin1. This suggested that there are likely prognostic and clinical applications of miR-296-5p in prostate cancer therapy [27]. In gastric cancer, $\mathrm{Li} \mathrm{T}$ et al. showed miR-296-5p over-expression significantly promoted GC cell growth and attenuated the CDX1-induced anti-growth effects by recurring cell cycle distribution and apoptotic status, whereas knockdown of miR-296-5p decreased GC cell growth [28], which is consistent with our result.

There are accumulating examples of circRNAs acting as miRNA sponges, thereby influencing the posttranscriptional actions of miRNAs as suppressors of the 


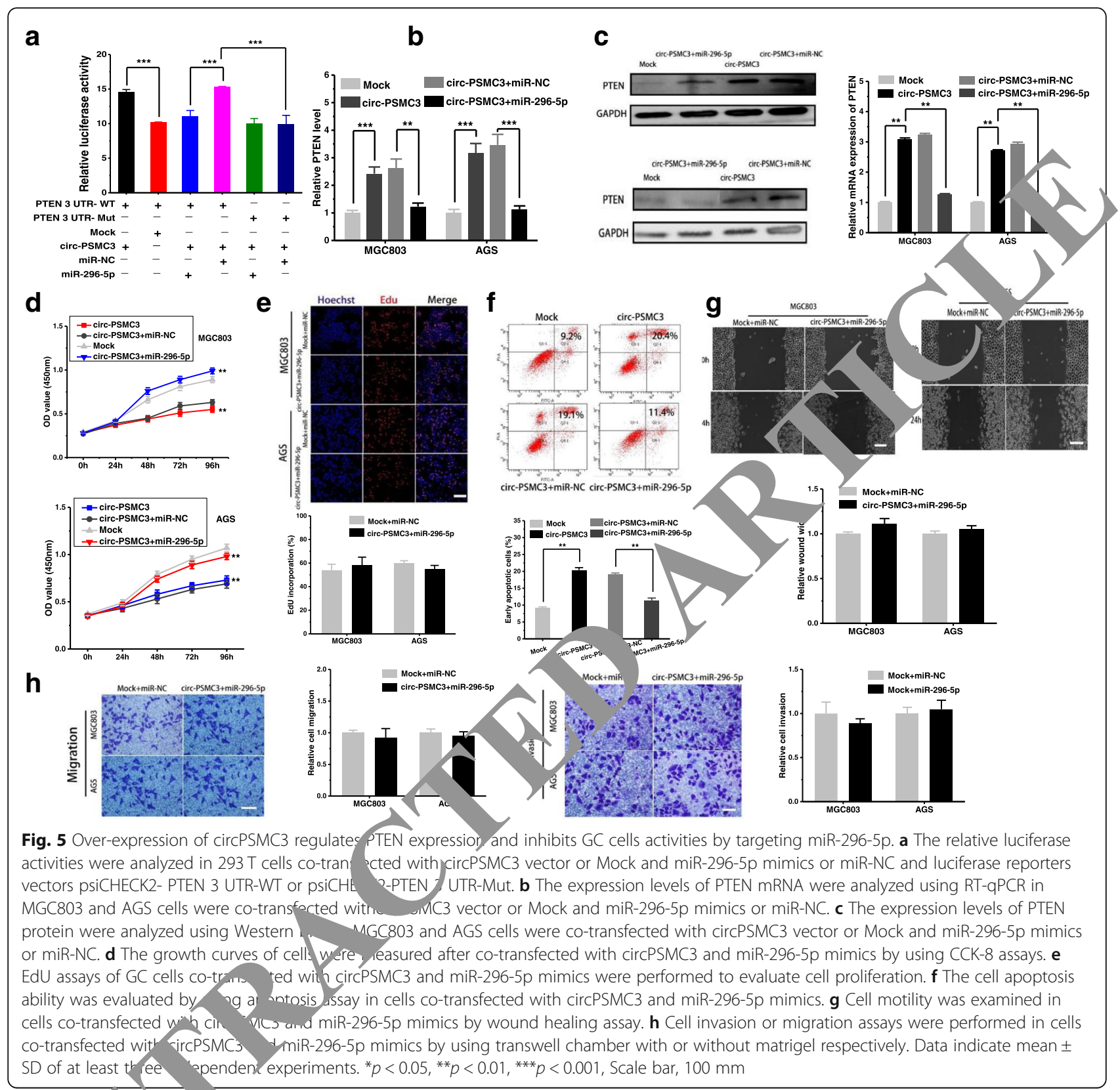

translat. d/Ar stability of target mRNAs [29, 30]. For ramp ci-ITCH (Itchy E3 ubiquitin protein lige) vas reported to sponge miR-7, miR-17, and $\mathrm{n} .214$, eading to the upregulation of ITCH and the inhlb on of WNT signaling in esophageal squamous cell carcinoma [31]. Li X found that hsa_circ_103809 could bind to miR-620 and negatively regulates miR-620 expression, further inhibiting the proliferation and invasion abilities of hepatocellular carcinoma cells [32]. In our research, we discovered that the over-expression of circPSMC3 could inhibit the proliferation, invasion and metastasis of GC cells. Furthermore, it can suppress the progression of GC by regulating miR-296-5p and PTEN expression. Functional inactivation of the tumor suppressor protein PTEN has been detected in multiple cases of GC, and already shown to be closely linked to the development, progression and prognosis of the disease. Inactivation of PTEN can be attributed to gene mutation, loss of heterozygosity, promoter hypermethylation, microRNA-mediated regulation of gene expression, and post-translational phosphorylation. PTEN is also involved in mechanisms regulating tumor resistance to chemotherapy [33]. Liu $\mathrm{S}$ et al. reported that low expression of PTEN and increased expression of miR-718 in GC tissues were both independent and unfavorable prognostic factors of GC. Up regulation of miR-718 


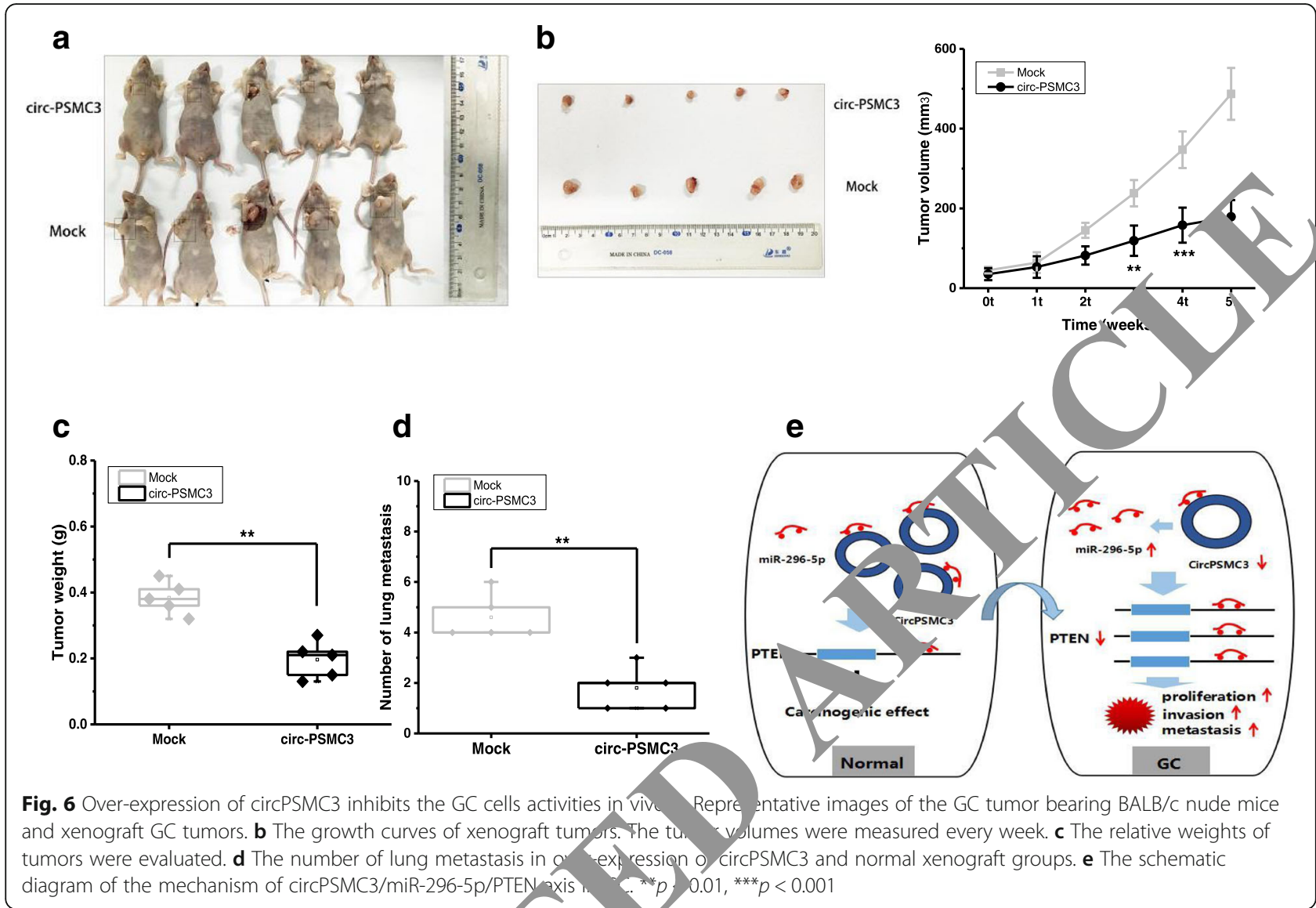

could increase PI3K/Akt signaling by airec down regulating PTEN, thus promoting th proliferatio, $A$ and invasion of gastric cancer cells [34 Liu $\mathrm{T}$ found that Circ-ZFR and PTEN were low- ressed whereas miR-107 and miR-130a were ${ }^{h}$-expressed in GC tissues and cells. There are targelea/cionships and interactions between $\mathrm{mi}$. $\mathrm{a} / \mathrm{m} \mathrm{R}-107$ and ZFR/PTEN. Circ-ZFR inhibits $G$ cel mronagation, cell cycle and promoted apoptosis b, sponging miR-107/miR-130a, while miR-107/ $\mathrm{R}-130 \mathrm{a}$, omoted GC cell propagation and prevented $a_{\text {a }}$ tosis through targeting PTEN. Circ-ZFP inhibited cell proliferation and facilitated apoptos. ir GC by sponging miR-130a/miR-107 and molatin $\mathrm{T}$ T N. Circ-ZFR curbed GC tumor growth nd a ected, 553 protein expression in vivo [35]. To our $k_{4}$ vieug, this is the first study to investigate the role of C. PSMC3 in gastric cancer. Not only that, this is also the first article to study the relationship between miR-296-5p and PTEN. These findings may bring light to the treatment of GC.

There are several limitations to the interpretation of our study results. Firstly, our study uses GC samples taken from an ethnically homogenous population and expects further sample size and more validation from different regions. Secondly, our study examines the ability of circPSMC3 to bind to miR-296-5p, but there may be other miRNAs that binds circPSMC3 to regulate the occurrence and progression of GC. Thirdly, whether circPSMC3 regulates the development of GC through other mechanisms such as protein binding requires further investigation. We hope that a follow-up study will elucidate a deeper understanding of the therapeutic potential of circPSMC3.

\section{Conclusion}

Our study identifies a new circular RNA, termed circPSMC3 that is down-regulated in tissues, corresponding plasmas from GC patients as well as GC cell lines and can act as a sponge of miRNA-296-5p to regulate the expression of PTEN. Our findings reveal a novel potential circulating biomarker for detection of GC.

\section{Additional file}

Additional file 1: Figure S1. (a) A total of 6 circRNAs based on the multiple fold difference in circRNA microarray and then were verified in a small sample of plasmas by using qRT-PCR. (b) SiRNA against circPSMC3 were designed to silence circPSMC3 level in BGC823 cells. (c) SiRNA against circPSMC3 were designed to silence circPSMC3 level in SGC7901 cells. (d) SiRNA\#1 against cirCPSMC3 were designed to silence circPSMC3 without influencing PSMC3 mRNA level in BGC823 and SGC7901cells. (e) 
The expression of circPSMC3 was evaluated in MGC803 and AGS cells transfected with circPSMC3 vector or Mock by using qRT-PCR. (f) The expression of PSMC3 mRNA was evaluated in MGC803 and AGS cells transfected with circPSMC3 vector or Mock by using qRT-PCR. (g) The expression of miR-296-5p was evaluated in MGC803 and AGS cells transfected with miR-296-5p inhibitor or miR-NC. (h) The expression of miR-296-5p was evaluated in MGC803 and AGS cells transfected with with miR-296-5p mimics or miR-NC. ${ }^{*} p<0.05,{ }^{* *} p<0.01,{ }^{* * *} p<0.001$. (DOCX $722 \mathrm{~kb}$ )

\section{Abbreviations}

CCK8: Cell counting kit-8; ceRNA: competitive endogenous RNA; circRNAs: Circular RNAs; EdU: 5-Ethynyl-20- deoxyuridine;

GAPDH: Glyceraldehyde-3-phosphate dehydrogenase; GC: Gastric cancer; GO: Gene oncology; IgG: immunoglobulin G; KEGG: Kyoto Encyclopedia of Genes and Genomes; miRNAs: MicroRNAs; PTEN: Phosphatase and Tensin Homolog; qRT-PCR: Quantitative reverse transcription polymerase reaction; RIP: RNA immunoprecipitation; RNA-FISH: RNA fluorescence in situ hybridization; ROC: Receiver-operating characteristic; WT: Wild type

\section{Acknowledgements}

We are grateful for participation and cooperation from the patients with gastric cancer.

\section{Funding}

This project was supported by Natural Science of Jiangsu Province (BK20151087) and the Development of Medical Science and Technology Foundation of Nanjing (Grant No.YKK17117) to Professor Hongyong Cao, the National Natural Science Foundation of China (Grant No.81572557 and 81872114) to Professor Shuli Zhao, and Natural Science Foundation of Nanjing medical university (Grant No.NMUB2018328) to Doctor Weiwei Tang.

\section{Availability of data and materials}

The datasets obtained and analyzed during the current study were avarable from the corresponding authors in a reasonable request.

\section{Authors' contributions}

There are 2 first authors in this manuscript and they have contributed to this project. DWR was responsible forcollecing specimen and their adjacent nontumorous tissues, ar wertas drafti manuscript. CL was responsiblefor designing and erforming the experiments. BZ was responsible for the manuscri language diting and data analysis. KF also contributed to performing pat the e periments, and data interpretation. Furthermore, we have three corres, suing authors in this manuscript. SLZ has contributed to da r.retation, editing and critical revision of the manuscript. WWT and HY $\mathrm{H}$ e contributed to study design and critical revision of th uscript SLZ, WWT, and HYC were also responsible for handling the vision and re-- abmission of revised manuscripts. All authors read and à, nal manuscript.

\section{Ethics approval ara isent to p. cicipate}

The human cance, tissu ced in this study were approved by the Ethics Committee of ...njing Firs sital, Nanjing Medical University.

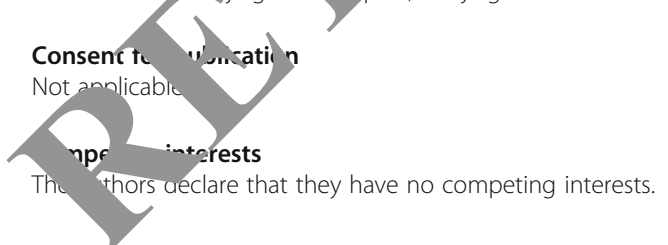

\section{Publisher's Note}

Springer Nature remains neutral with regard to jurisdictional claims in published maps and institutional affiliations.

\section{Author details}

'Department of General Surgery, Nanjing First Hospital, Nanjing Medical University, Nanjing, Jiangsu, China. ${ }^{2}$ Michael G. DeGroote School of Medicine, McMaster University, Hamilton, Ontario, Canada. ${ }^{3}$ Department of General Clinical Research Center, Nanjing First Hospital, Nanjing Medical University, Nanjing, Jiangsu, China.
Received: 29 September 2018 Accepted: 13 February 2019 Published online: 18 February 2019

\section{References}

1. Ferlay J, Soerjomataram I, Dikshit R, et al. Cancer incidence and mortality worldwide: sources, methods and major patterns in Globocan. Int J Cancer. 2015:136:E359-86.

2. Valastyan S, Weinberg RA. Tumor Metastasis: Molecular insigh evolving paradigms. Cell. 2011;147:275-92.

3. Uemura $\mathrm{N}$, Okamoto $\mathrm{S}$, Yamamoto $\mathrm{S}$, et al. Helicobacter pylori infe the development of gastric cancer. N Engl J Med. 20 ·345:784-9

4. Zhuang M, Gao W, Xu J, et al. The long non-coding $h$, -19 -deril d' miR675 modulates human gastric cancer cell proliferation by retir. $y$ tumor suppressor RUNX1. Biochem Biophys Res Cr nmun. 2014;44، 15-22.

5. Ito K, Chuang LS, Ito T, et al. Loss of Runx3 is key event in inducing precancerous state of the stomach. Ge troen logy. 201 1;140:1536-1546e8.

6. Wilusz JE, Sharp PA. A circuitous rou non, RNA. Science. 2013; 340:440e441.

7. Szabo L, Morey R, Palpant al. Statistic based splicing detection reveals neural enrichmer ana ve-specific induction of circular RNA during human fetal development. nome Biol. 2015;16:126.

8. Salzman J, Chen R, MN, et al, orown, cell-type specific features of circular RNA ey ssion il oS Genet. 2013;9:e1003777.

9. Li J, Yang J, Zhou _, Ircular RNAs in cancer: novel insights into origins, properties, fu ns and implications. Am J Cancer Res. 2015;5:472-80.

10. Hsiao K- Y-C, Gup, SK, et al. Non-coding effects of circular RNA CCDC66 rom son cancer growth and metastasis. Cancer Res. 2017; 77:2339-5

11. Du WW, Yar a W, Liu E, et al. Foxo3 circular RNA retards cell cycle gression via forming ternary complexes with p21 and CDK2. Nucleic s Res. 2016:44:2846e2858.

Ha sen TB, Jensen TI, Clausen BH, et al. Natural RNA circles function as cient microRNA sponges. Nature. 2013:495:384e388.

13. Holdt LM, Stahringer A, Sass K, et al. Circular non-coding RNA ANRIL modulates ribosomal RNA maturation and atherosclerosis in humans. Nat Commun. 2016;7:12429.

14. Han D, Li J, Wang H, et al. Circular RNA circMTO1 acts as the sponge of MicroRNA-9 to suppress hepatocellular carcinoma progression. Hepatology. 2017:66:1151-64.

15. Zhong Z, Huang M, Lv M, et al. Circular RNA MYLK as a competing endogenous RNA promotes bladder cancer progression through modulating VEGFANEGFR2 signaling pathway. Cancer Letters. 2017;403: 305-17.

16. Thomson DW, Dinger ME. Endogenous microRNA sponges: evidence and controversy. Nat Rev Genet. 2016;17:272-83.

17. Memczak S, Jens M, Elefsinioti $A$, et al. Circular RNAs are a large class of animal RNAs with regulatory potency. Nature. 2013;495:333-8.

18. Chen L, Zhang S, Wu J, et al. circRNA 100290 plays a role in oral cancer by functioning as a sponge of the miR-29 family. Oncogene. 2017;36:4551-61.

19. Wei $X, \mathrm{Li} \mathrm{H}$, Yang J, et al. Circular RNA profiling reveals an abundant circLMO7 that regulates myoblasts differentiation and survival by sponging miR-378a-3p. Cell Death Dis. 2017:8(8):e3153.

20. Li Y, Zheng F, Xiao X, et al. CircHIPK3 sponges miR-558 to suppress heparanase expression in bladder cancer cells. EMBO Rep. 2017:18:1646-59.

21. Sheng JQ, Liu L, Wang MR, et al. Circular RNAs in digestive system cancer: potential biomarkers and therapeutic targets. Am J Cancer Res. 2018:8:1142-56

22. Fang Y. Circular RNAs as novel biomarkers with regulatory potency in human diseases. Future Sci OA. 2018;4:FSO314.

23. Kristensen L, Hansen S, et al. Circular RNAs in cancer: opportunities and challenges in the field. Oncogene. 2017;37:555-65.

24. Xie $Y$, Shao $Y$, Sun $W$, et al. Downregulated expression of hsa circ 0074362 in gastric cancer and its potential diagnostic values. Biomark Med. 2018;12:11-20.

25. Lee $\mathrm{H}$, Shin $\mathrm{CH}$, Kim HR, et al. MicroRNA-296-5p promotes invasiveness through downregulation of nerve growth factor receptor and Caspase-8. Mol Cells. 2017:40:254-61.

26. Maia D, de Carvalho AC, Horst MA, et al. Expression of miR-296-5p as predictive marker for radiotherapy resistance in early-stage laryngeal carcinoma. J Transl Med. 2015;13:262. 
27. Lee KH, Lin FC, Hsu Tl, et al. MicroRNA-296-5p (miR-296-5p) functions as a tumor suppressor in prostate cancer by directly targeting Pin1. Biochim Biophys Acta. 1843;2014:2055-66.

28. Li T, Lu YY, Zhao XD, et al. MicroRNA-296-5p increases proliferation in gastric cancer through repression of caudal-related homeobox 1. Oncogene. 2014;33:783-93.

29. Qu D, Yan B, Xin R, Ma T, et al. A novel circular RNA hsa_circ_0020123 exerts oncogenic properties through suppression of miR-144 in non-small cell lung cancer. Am J Cancer Res. 2018;8:1387-402.

30. Xiao T, Xue J, Shi M, et al. Circ008913, via miR-889 regulation of DAB2IP/ ZEB1, is involved in the arsenite-induced acquisition of CSC-like properties by human keratinocytes in carcinogenesis. Metallomics. 2018;10:1328-38.

31. Yang C, Yuan W, Yang X, et al. Circular RNA circ-ITCH inhibits bladder cancer progression by sponging miR-17/miR-224 and regulating p21, PTEN expression. Mol Cancer. 2018:17:19.

32. Li X, Shen M. Circular RNA hsa_circ_103809 suppresses hepatocellular carcinoma proliferation and invasion by sponging miR-620. Eur Rev Med Pharmacol Sci. 2019;23:555-66.

33. Xu WT, Yang Z, Lu NH, et al. Roles of PTEN (phosphatase and Tensin homolog) in gastric cancer development and progression. Asian Pac Cancer Prev. 2014;15:17-24.

34. Liu S, Tian Y, Zhu C, et al. High miR-718 suppresses phosphatase and Tensin homolog (PTEN) expression and correlates to unfavorable prognosis in gastric Cancer. Med Sci Monit. 2018;24:5840-50.

35. Liu T, Liu S, Xu Y, et al. Circular RNA-ZFR inhibited cell proliferation and promoted apoptosis in gastric Cancer by sponging miR-130a/miR-107 and modulating PTEN. Cancer Res Treat. 2018;50(4):1396-417.
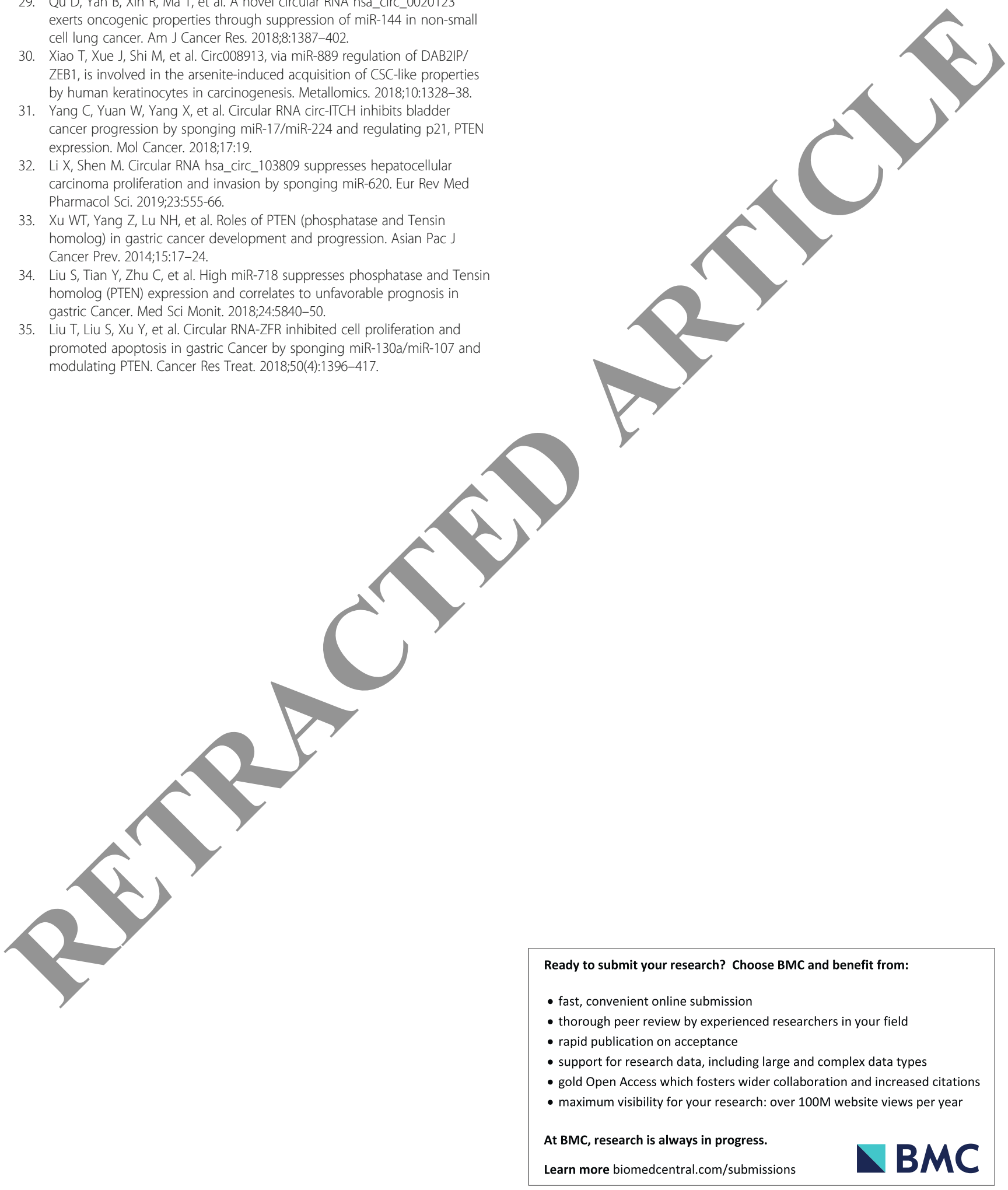